doi: $10.2306 /$ scienceasia1513-1874.2012.38.196

\title{
Regularity criterion for weak solutions to the 3D Boussinesq equations
}

\author{
Yin-Xia Wang ${ }^{\mathrm{a}, *}$, Huimin Gao ${ }^{\mathrm{b}}$ \\ a College of Mathematics and Information Science, \\ North China University of Water Resources and Electric Power, Zhengzhou 450011, China \\ b School of Mathematics and Statistics, Henan University of Science and Technology, Luoyang 471003, China
}

*Corresponding author, e-mail: yinxia117@126.com

Received 18 Feb 2012

Accepted 22 May 2012

ABSTRACT: In this paper, a regularity criterion for the 3D Boussinesq equations is investigated. We prove that for some $T>0$ if $\int_{0}^{T}\left\|u_{z}\right\|_{L^{\alpha}}^{\beta} \mathrm{d} t<\infty$, where $3 / \alpha+2 / \beta \leqslant 1$ and $\alpha \geqslant 3$, then the solution $(u, \theta)$ can be extended smoothly beyond $t=T$. The derivative $u_{z}$ can be replaced by any directional derivative of $u$.

KEYWORDS: global regularity, energy estimate

\section{INTRODUCTION}

In the paper we investigate the initial value problem for the Boussinesq equations in $\mathbb{R}^{3}$

$$
\begin{array}{r}
\partial_{t} u-\mu \Delta u+u \cdot \nabla u+\nabla \Pi=\theta \hat{z}, \\
\partial_{t} \theta-\nu \Delta \theta+u \cdot \nabla \theta=0, \\
\nabla \cdot u=0
\end{array}
$$

with the initial value

$$
t=0: \quad u=u_{0}(x), \theta=\theta_{0}(x) .
$$

where $u$ is the velocity, $\Pi$ is the pressure, $\theta$ is the temperature, $\mu$ is the viscosity, $\nu$ is called the molecular diffusivity, and $\hat{z}=(0,0,1)^{\mathrm{T}}$.

The Boussinesq equations are of relevance in studying a number of models coming from atmospheric or oceanographic turbulence where rotation and stratification play an important role ${ }^{1}$. The scalar function $\theta$ may for instance represent temperature variation in a gravity field, and $\theta \hat{z}$ the buoyancy force. Fundamental mathematical issues such as the global regularity of their solutions have generated extensive research and many interesting results have been obtained; see Refs. 2-4 for regularity criteria and see Refs. 5-7 for blow up criteria.

If $\theta=0$, then (1) reduce to the Navier-Stokes equations. Besides their physical applications, the Navier-Stokes equations are also mathematically significant. Leray $^{8}$ and Hopf ${ }^{9}$ constructed weak solutions to the Navier-Stokes equations. The solution is called the Leray-Hopf weak solution. From then on, much effort has been devoted to establishing the global existence and uniqueness of smooth solutions to the Navier-Stokes equations. Lots of regularity criteria of the weak solutions have been proposed and many interesting results have been established (e.g., regularity criteria via the velocity ${ }^{10-12}$, via the derivative of the velocity in one or two direction ${ }^{13-15}$, and via the pressure ${ }^{16-18}$ ). Logarithmically improved regularity criteria of weak solutions were established in Refs. 19, 20.

The purpose of this paper is to establish the regularity criteria of weak solutions to (1) and (2) via the derivative of the velocity in one direction. We first give the definition of a weak solution.

Definition 1 Let $u_{0}, \theta_{0} \in L^{2}\left(\mathbb{R}^{3}\right)$ with $\nabla \cdot u_{0}=0$. Then $(u, \theta)$ is called a weak solution of (1) and (2) if the following conditions are satisfied.

(i) $u, \theta \in L^{2}\left(0, T ; H^{1}\left(\mathbb{R}^{3}\right)\right) \bigcap C_{w}\left(0, T ; L^{2}\left(\mathbb{R}^{3}\right)\right)$ with $\nabla \cdot u=0$ in the sense of distribution.

(ii) For any $\varphi, \psi \in C_{0}^{\infty}\left([0, T) \times \mathbb{R}^{3}\right)$ with $\nabla \cdot \varphi=0$ and $\varphi(T, \cdot)=0, \psi(T, \cdot)=0$, there holds

$$
\begin{array}{r}
\int_{0}^{T} \int_{\mathbb{R}^{3}}\left(-u \varphi_{t}+\mu \nabla u \cdot \nabla \varphi-u(u \cdot \nabla) \varphi\right) \mathrm{d} r \mathrm{~d} t \\
=\int_{0}^{T} \int_{\mathbb{R}^{3}} \theta \hat{z} \cdot \varphi \mathrm{d} r \mathrm{~d} t+\int_{\mathbb{R}^{3}} u_{0} \varphi(0, \cdot) \mathrm{d} r \\
\int_{0}^{T} \int_{\mathbb{R}^{3}}\left(-\theta \psi_{t}+\nu \nabla \theta \cdot \nabla \psi-\theta u \cdot \nabla \psi\right) \mathrm{d} r \mathrm{~d} t \\
=\int_{\mathbb{R}^{3}} \theta_{0} \psi(0, \cdot) \mathrm{d} r .
\end{array}
$$


where $r=(x, y, z)$.

(iii) For almost all $t_{0} \in[0, T]$ including $t_{0}=0$ and for all $t \geqslant t_{0}$,

$$
\begin{aligned}
\|u(t)\|_{L^{2}}^{2}+ & 2 \mu \int_{t_{0}}^{t}\|\nabla u(\tau)\|_{L^{2}}^{2} \mathrm{~d} \tau \\
& \leqslant\left\|u\left(t_{0}\right)\right\|_{L^{2}}^{2}+2 \int_{t_{0}}^{t} \int_{\mathbb{R}^{3}} \theta \hat{z} \cdot u \mathrm{~d} r \mathrm{~d} \tau, \\
\|\theta(t)\|_{L^{2}}^{2} & +2 \nu \int_{t_{0}}^{t}\|\nabla \theta(\tau)\|_{L^{2}}^{2} \mathrm{~d} \tau \leqslant\left\|\theta\left(t_{0}\right)\right\|_{L^{2}}^{2} .
\end{aligned}
$$

For simplicity, we take $\mu=\nu=1$. Our main result is as follows.

Theorem 1 Let $u_{0}, \theta_{0} \in H^{1}\left(\mathbb{R}^{3}\right)$ with $\nabla \cdot u_{0}=0$. Assume that $(u, \theta)$ is a weak solution to (1) and (2) on some interval $[0, T]$. If

$$
\Theta(T) \equiv \int_{0}^{T}\left\|u_{z}\right\|_{L^{\alpha}}^{\beta} \mathrm{d} t<\infty,
$$

where $3 / \alpha+2 / \beta \leqslant 1, \alpha \geqslant 3$, then the solution $(u, \theta)$ can be extended smoothly beyond $t=T$.

\section{SOME USEFUL LEMMMAS}

In order to prove our main result, we need the following Lemma, which may be found in Refs. 21-23.

Lemma 1 Assume that $\mu, \lambda, \iota \in \mathbb{R}$ and satisfy

$$
1 \leqslant \iota, \lambda<\infty, \frac{1}{\iota}+\frac{2}{\lambda}>1,1+\frac{3}{\vartheta}=\frac{1}{\iota}+\frac{2}{\lambda} .
$$

Assume that $f \in H^{1}\left(\mathbb{R}^{3}\right), f_{x}, f_{y} \in L^{\lambda}\left(\mathbb{R}^{3}\right)$ and $f_{z} \in$ $L^{\iota}\left(\mathbb{R}^{3}\right)$. Then there exists a positive constant such that

$$
\|f\|_{L^{\vartheta}} \leqslant C\left\|f_{x}\right\|_{L^{\lambda}}^{\frac{1}{3}}\left\|f_{y}\right\|_{L^{\lambda}}^{\frac{1}{3}}\left\|f_{z}\right\|_{L^{\iota}}^{\frac{1}{3}} .
$$

In particular, when $\lambda=2$, there exists a positive constant $C=C(\iota)$ such that

$$
\|f\|_{L^{3 \iota}} \leqslant C\left\|f_{x}\right\|_{L^{2}}^{\frac{1}{3}}\left\|f_{y}\right\|_{L^{2}}^{\frac{1}{3}}\left\|f_{z}\right\|_{L^{\mu}}^{\frac{1}{3}} .
$$

which holds for any $f \in H^{1}\left(\mathbb{R}^{3}\right)$ and $f_{z} \in L^{\mu}\left(\mathbb{R}^{3}\right)$ with $1 \leqslant \mu<\infty$.

Using Lemma 1, we obtain the following Lemma (see also Ref. 22).

Lemma 2 Let $2 \leqslant q \leqslant 6$ and assume that $f \in$ $H^{1}\left(\mathbb{R}^{3}\right)$. Then there exists a positive constant $C=$ $C(q)$ such that

$$
\|f\|_{L^{q}} \leqslant C\|f\|_{L^{2}}^{\frac{6-q}{2 q}}\left\|\partial_{x} f\right\|_{L^{2}}^{\frac{q-2}{2 q}}\left\|\partial_{y} f\right\|_{L^{2}}^{\frac{q-2}{2 q}}\left\|\partial_{z} f\right\|_{L^{2}}^{\frac{q-2}{2 q}}
$$

\section{PROOF OF MAIN RESULT}

For given initial data $u_{0}, \theta_{0} \in H^{1}\left(\mathbb{R}^{3}\right)$, the weak solution is the same as the local strong solution $(u, \theta)$ in a local interval $(0, T)$ as in the discussion of the NavierStokes equations. Thus Theorem 1 is reduced to establishing a priori estimates uniformly in $(0, T)$ for strong solutions. With the use of the a priori estimates, the local strong solution $(u, \theta)$ can be continuously extended to $t=T$ by a standard process to obtain global regularity of the weak solution. Therefore, we assume that the solution $(u, \theta)$ is sufficiently smooth on $(0, T)$.

Multiplying (1b) by $\theta$ and integrating with respect to $r=(x, y, z)$ on $\mathbb{R}^{3}$, and using integration by parts, we obtain

$$
\frac{1}{2} \frac{\mathrm{d}}{\mathrm{d} t}\|\theta(t)\|_{L^{2}}^{2}+\|\nabla \theta(t)\|_{L^{2}}^{2}=0 .
$$

Similarly, we get

$$
\frac{1}{2} \frac{\mathrm{d}}{\mathrm{d} t}\|u(t)\|_{L^{2}}^{2}+\|\nabla u(t)\|_{L^{2}}^{2} \int_{\mathbb{R}^{3}}(\theta \hat{z}) u \mathrm{~d} r .
$$

Summing (7) and (8) and using Cauchy's inequality, we deduce that

$$
\begin{gathered}
\frac{1}{2} \frac{\mathrm{d}}{\mathrm{d} t}\left(\|u(t)\|_{L^{2}}^{2}+\|\theta(t)\|_{L^{2}}^{2}\right)+\|\nabla u(t)\|_{L^{2}}^{2}+\|\nabla \theta(t)\|_{L^{2}}^{2} \\
\leqslant \frac{1}{2}\left(\|u(t)\|_{L^{2}}^{2}+\|\theta(t)\|_{L^{2}}^{2}\right) . \quad \text { (9) }
\end{gathered}
$$

It follows from (9) and Gronwall's inequality that

$$
\begin{aligned}
\|u(t)\|_{L^{2}}^{2}+\|\theta(t)\|_{L^{2}}^{2} & +2 \int_{0}^{t}\left(\|\nabla u\|_{L^{2}}^{2}+\|\nabla \theta\|_{L^{2}}^{2}\right) \mathrm{d} \tau \\
& \leqslant C\left(\left\|u_{0}\right\|_{L^{2}}^{2}+\left\|\theta_{0}\right\|_{L^{2}}^{2}\right) .
\end{aligned}
$$

Differentiating (1a) and (1b) with respect to $z$, we obtain

$$
\begin{gathered}
\partial_{t} u_{z}-\Delta u_{z}+u_{z} \cdot \nabla u+u \cdot \nabla u_{z}+\nabla \Pi_{z}=(\theta \hat{z})_{z}, \\
\partial_{t} \theta_{z}-\Delta \theta_{z}+u_{z} \cdot \nabla \theta+u \cdot \nabla \theta_{z}=0 .
\end{gathered}
$$

Taking the inner product of $u_{z}$ with (11a) and using integration by parts yields

$$
\begin{aligned}
& \frac{1}{2} \frac{\mathrm{d}}{\mathrm{d} t}\left\|u_{z}(t)\right\|_{L^{2}}^{2}+\left\|\nabla u_{z}(t)\right\|_{L^{2}}^{2} \\
& \quad=-\int_{\mathbb{R}^{3}} u_{z} \cdot \nabla u \cdot u_{z} \mathrm{~d} r+\int_{\mathbb{R}^{3}}(\theta \hat{z})_{z} \cdot u_{z} \mathrm{~d} r
\end{aligned}
$$

Similarly, we get

$\frac{1}{2} \frac{\mathrm{d}}{\mathrm{d} t}\left\|\theta_{z}(t)\right\|_{L^{2}}^{2}+\left\|\nabla \theta_{z}(t)\right\|_{L^{2}}^{2}=-\int_{\mathbb{R}^{3}} u_{z} \cdot \nabla \theta \cdot \theta_{z} \mathrm{~d} r$. 
Combining (12) and (13) yields

$$
\begin{gathered}
\frac{1}{2} \frac{\mathrm{d}}{\mathrm{d} t}\left(\left\|u_{z}(t)\right\|_{L^{2}}^{2}+\left\|\theta_{z}(t)\right\|_{L^{2}}^{2}\right) \\
+\left\|\nabla u_{z}(t)\right\|_{L^{2}}^{2}+\left\|\nabla \theta_{z}(t)\right\|_{L^{2}}^{2} \\
=-\int_{\mathbb{R}^{3}} u_{z} \cdot \nabla u \cdot u_{z} \mathrm{~d} r+\int_{\mathbb{R}^{3}}(\theta \hat{z})_{z} \cdot u_{z} \mathrm{~d} r \\
\quad-\int_{\mathbb{R}^{3}} u_{z} \cdot \nabla \theta \cdot \theta_{z} \mathrm{~d} r \\
\triangleq I_{1}+I_{2}+I_{3} .
\end{gathered}
$$

In what follows, we estimate the $I_{j}$. By integration by parts and Hölder's inequality, we obtain

$$
I_{1} \leqslant C\left\|\nabla u_{z}\right\|_{L^{2}}\left\|u_{z}\right\|_{L^{\sigma}}\|u\|_{L^{3 \alpha}},
$$

where

$$
\frac{1}{\sigma}+\frac{1}{3 \alpha}=\frac{1}{2}, \quad 2 \leqslant \sigma \leqslant 6 .
$$

It follows from the interpolating inequality that

$$
\left\|u_{z}\right\|_{L^{\sigma}} \leqslant C\left\|u_{z}\right\|_{L^{2}}^{1-3\left(\frac{1}{2}-\frac{1}{\sigma}\right)}\left\|\nabla u_{z}\right\|_{L^{2}}^{3\left(\frac{1}{2}-\frac{1}{\sigma}\right)} .
$$

From (5), we get

$$
\begin{aligned}
I_{1} \leqslant C & \left\|\nabla u_{z}\right\|_{L^{2}}\left\|u_{z}\right\|_{L^{2}}^{1-3\left(\frac{1}{2}-\frac{1}{\sigma}\right)} \\
& \times\left\|\nabla u_{z}\right\|_{L^{2}}^{3\left(\frac{1}{2}-\frac{1}{\sigma}\right)}\|\nabla u\|_{L^{2}}^{\frac{2}{3}}\left\|u_{z}\right\|_{L^{\alpha}}^{\frac{1}{3}} \\
\leqslant & C\left\|\nabla u_{z}\right\|_{L^{2}}^{1+3\left(\frac{1}{2}-\frac{1}{\sigma}\right)}\left\|u_{z}\right\|_{L^{2}}^{1-3\left(\frac{1}{2}-\frac{1}{\sigma}\right)} \\
& \times\|\nabla u\|_{L^{2}}^{\frac{2}{3}}\left\|u_{z}\right\|_{L^{\alpha}}^{\frac{1}{3}} \\
\leqslant & \frac{1}{2}\left\|\nabla u_{z}\right\|_{L^{2}}^{2}+C\left\|u_{z}\right\|_{L^{2}}^{2}\|\nabla u\|_{L^{2}}^{2 q}\left\|u_{z}\right\|_{L^{\alpha}}^{q},
\end{aligned}
$$

where

$$
q=\frac{2}{3-9\left(\frac{1}{2}-\frac{1}{\sigma}\right)}=\frac{2}{3\left(1-\frac{1}{\alpha}\right)} .
$$

When $\alpha \geqslant 3$, we have $2 q \leqslant 2$ and application of Young's inequality yields

$$
I_{1} \leqslant \frac{1}{2}\left\|\nabla u_{z}\right\|_{L^{2}}^{2}+C\left\|u_{z}\right\|_{L^{2}}^{2}\left(\|\nabla u\|_{L^{2}}^{2}+\left\|u_{z}\right\|_{L^{\alpha}}^{\delta}\right),
$$

where

$$
\frac{3}{\alpha}+\frac{2}{\delta}=1
$$

By Cauchy's inequality, we have

$$
I_{2} \leqslant \frac{1}{2}\left(\left\|u_{z}\right\|_{L^{2}}^{2}+\left\|\theta_{z}\right\|_{L^{2}}^{2}\right) .
$$

From Hölder's inequality, we obtain

$$
\begin{aligned}
I_{3} & \leqslant C\|\nabla \theta\|_{L^{2}}\left\|\theta_{z}\right\|_{L^{\frac{2 \alpha}{\alpha-2}}}\left\|u_{z}\right\|_{L^{\alpha}} \\
& \leqslant C\|\nabla \theta\|_{L^{2}}\left\|u_{z}\right\|_{L^{\alpha}}\left\|\theta_{z}\right\|_{L^{2}}^{1-\frac{3}{\alpha}}\left\|\nabla \theta_{z}\right\|_{L^{2}}^{\frac{3}{\alpha}} \\
& \leqslant \frac{1}{2}\left\|\nabla \theta_{z}\right\|_{L^{2}}^{2}+\|\nabla \theta\|_{L^{2}}^{\frac{2 \alpha}{2 \alpha-3}}\left\|u_{z}\right\|_{L^{\alpha}}^{\frac{2 \alpha}{2 \alpha-3}}\left\|\theta_{z}\right\|_{L^{2}}^{\frac{2 \alpha-6}{2 \alpha-3}} \\
& \leqslant \frac{1}{2}\left\|\nabla \theta_{z}\right\|_{L^{2}}^{2}+C\left(\|\nabla \theta\|_{L^{2}}^{2}+\left\|u_{z}\right\|_{L^{\alpha}}^{\delta}\right)\left\|\theta_{z}\right\|_{L^{2}}^{\frac{2 \alpha-6}{2 \alpha-3}}
\end{aligned}
$$

Combining (14)-(17) yields

$$
\begin{gathered}
\frac{\mathrm{d}}{\mathrm{d} t}\left(\left\|u_{z}\right\|_{L^{2}}^{2}+\left\|\theta_{z}\right\|_{L^{2}}^{2}\right)+\left\|\nabla u_{z}\right\|_{L^{2}}^{2}+\left\|\nabla \theta_{z}\right\|_{L^{2}}^{2} \\
\leqslant C\left(\left\|u_{z}\right\|_{L^{2}}^{2}+\left\|\theta_{z}\right\|_{L^{2}}^{2}\right)\left(\|\nabla u\|_{L^{2}}^{2}+\left\|u_{z}\right\|_{L^{\alpha}}^{\delta}+1\right) \\
+C\left(\|\nabla \theta\|_{L^{2}}^{2}+\left\|u_{z}\right\|_{L^{\alpha}}^{\delta}\right)\left\|\theta_{z}\right\|_{L^{2}}^{\frac{2 \alpha-6}{2 \alpha-3}}
\end{gathered}
$$

From Gronwall's inequality, we get

$$
\begin{gathered}
\left\|u_{z}\right\|_{L^{2}}^{2}+\left\|\theta_{z}\right\|_{L^{2}}^{2}+s \int_{0}^{t}\left\|\nabla u_{z}\right\|_{L^{2}}^{2} \mathrm{~d} \tau \\
+\int_{0}^{t}\left\|\nabla \theta_{z}\right\|_{L^{2}}^{2} \mathrm{~d} \tau \\
\leqslant C \mathrm{e}^{\left(\left\|u_{0}\right\|_{L^{2}}^{2}+\left\|\theta_{0}\right\|_{L^{2}}^{2}\right)} \mathrm{e}^{\Theta(t)}\left[\left\|u_{0}\right\|_{H^{1}}^{2}+\left\|\theta_{0}\right\|_{H^{1}}^{2}\right. \\
\left.C\left(\left\|u_{0}\right\|_{L^{2}}^{2}+\left\|\theta_{0}\right\|_{L^{2}}^{2}+\Theta(t)\right)^{\frac{2 \alpha-3}{\alpha}}\right] .
\end{gathered}
$$

Multiplying (1a) by $-\Delta u$ and integrating with respect to $x$ on $\mathbb{R}^{3}$, then using integration by parts, we obtain

$$
\begin{aligned}
& \frac{1}{2} \frac{\mathrm{d}}{\mathrm{d} t}\|\nabla u(t)\|_{L^{2}}^{2}+\|\Delta u\|_{L^{2}}^{2} \\
& \quad=\int_{\mathbb{R}^{3}} u \cdot \nabla u \cdot \Delta u \mathrm{~d} r-\int_{\mathbb{R}^{3}}(\theta \hat{z}) \cdot \Delta u \mathrm{~d} r .
\end{aligned}
$$

Similarly, we get

$$
\frac{1}{2} \frac{\mathrm{d}}{\mathrm{d} t}\|\nabla \theta(t)\|_{L^{2}}^{2}+\|\Delta \theta\|_{L^{2}}^{2}=\int_{\mathbb{R}^{3}} u \cdot \nabla \theta \cdot \Delta \theta \mathrm{d} r .
$$

Collecting (19) and (20) yields

$$
\begin{gathered}
\frac{1}{2} \frac{\mathrm{d}}{\mathrm{d} t}\left(\|\nabla u(t)\|_{L^{2}}^{2}+\|\nabla \theta(t)\|_{L^{2}}^{2}\right)+\|\Delta u\|_{L^{2}}^{2}+\|\Delta \theta\|_{L^{2}}^{2} \\
=\int_{\mathbb{R}^{3}} u \cdot \nabla u \cdot \Delta u \mathrm{~d} r-\int_{\mathbb{R}^{3}}(\theta \hat{z}) \cdot \Delta u \mathrm{~d} r \\
\quad+\int_{\mathbb{R}^{3}} u \cdot \nabla \theta \cdot \Delta \theta \mathrm{d} r \\
\triangleq J_{1}+J_{2}+J_{3}
\end{gathered}
$$

In what follows, we estimate the $J_{i}$. By (6) and 
Young's inequality, we deduce that

$$
\begin{aligned}
J_{1} \leqslant & C\|\nabla u\|_{L^{3}}^{3} \\
\leqslant & C\|\nabla u\|_{L^{2}}^{\frac{3}{2}}\left\|\nabla_{\perp} \nabla u\right\|_{L^{2}}\left\|\nabla u_{z}\right\|_{L^{2}}^{\frac{1}{2}} \\
\leqslant & \frac{1}{4}\|\nabla \perp \nabla u\|_{L^{2}}^{2}+C\|\nabla u\|_{L^{2}}^{3}\left\|\nabla u_{z}\right\|_{L^{2}} \\
\leqslant & \frac{1}{4}\left\|\nabla_{\perp} \nabla u\right\|_{L^{2}}^{2}+C\left(\|\nabla u\|_{L^{2}}^{2}+\left\|\nabla u_{z}\right\|_{L^{2}}^{2}\right) \\
& \quad \times\|\nabla u\|_{L^{2}}^{2}
\end{aligned}
$$

where $\nabla_{\perp}=\left(\partial_{x}, \partial_{y}\right)$.

Using integration by parts and Cauchy's inequality, we have

$$
J_{2} \leqslant \frac{1}{2}\left(\|\nabla u\|_{L^{2}}^{2}+\|\nabla \theta\|_{L^{2}}^{2}\right) .
$$

By (6) and Young's inequality, we have

$$
\begin{aligned}
J_{3} \leqslant & \|\nabla u\|_{L^{3}}\|\nabla \theta\|_{L^{3}}^{2} \\
\leqslant & C\|\nabla u\|_{L^{2}}^{\frac{1}{2}}\left\|\nabla_{\perp} \nabla u\right\|_{L^{2}}^{\frac{1}{3}}\left\|\nabla u_{z}\right\|_{L^{2}}^{\frac{1}{6}} \\
& \times\|\nabla \theta\|_{L^{2}}\left\|\nabla_{\perp} \nabla \theta\right\|_{L^{2}}^{\frac{2}{3}}\left\|\nabla \theta_{z}\right\|_{L^{2}}^{\frac{1}{3}} \\
\leqslant & \frac{1}{4}\left\|\nabla_{\perp} \nabla u\right\|_{L^{2}}^{2}+C\|\nabla u\|_{L^{2}}^{\frac{3}{5}}\left\|\nabla u_{z}\right\|_{L^{2}}^{\frac{1}{5}} \\
& \times\|\nabla \theta\|_{L^{2}}^{\frac{6}{5}}\left\|\nabla \nabla_{\perp} \theta\right\|_{L^{2}}^{\frac{4}{5}}\left\|\nabla \theta_{z}\right\|_{L^{2}}^{\frac{2}{5}} \\
\leqslant & \frac{1}{4}\left\|\nabla_{\perp} \nabla u\right\|_{L^{2}}^{2}+\frac{1}{2}\left\|\nabla_{\perp} \nabla \theta\right\|_{L^{2}}^{2} \\
& +C\|\nabla u\|_{L^{2}}\left\|\nabla u_{z}\right\|_{L^{2}}^{\frac{1}{3}}\|\nabla \theta\|_{L^{2}}^{2}\left\|\nabla \theta_{z}\right\|_{L^{2}}^{\frac{2}{3}} \\
\leqslant & \frac{1}{4}\left\|\nabla_{\perp} \nabla u\right\|_{L^{2}}^{2}+\frac{1}{2}\left\|\nabla_{\perp} \nabla \theta\right\|_{L^{2}}^{2}+C\|\nabla \theta\|_{L^{2}}^{2} \\
& \times\left(\|\nabla u\|_{L^{2}}^{2}+\left\|\nabla u_{z}\right\|_{L^{2}}^{2}+\left\|\nabla \theta_{z}\right\|_{L^{2}}^{2}\right) . \quad(24)
\end{aligned}
$$

Combining (21)-(24) yields

$$
\begin{aligned}
& \frac{\mathrm{d}}{\mathrm{d} t}\left(\|\nabla u(t)\|_{L^{2}}^{2}+\|\nabla \theta(t)\|_{L^{2}}^{2}\right)+\|\Delta u\|_{L^{2}}^{2}+\|\Delta \theta\|_{L^{2}}^{2} \\
& \leqslant C\left(\|\nabla u\|_{L^{2}}^{2}+\|\nabla \theta\|_{L^{2}}^{2}\right)\left(\|\nabla u\|_{L^{2}}^{2}\right. \\
& \left.+\left\|\nabla u_{z}\right\|_{L^{2}}^{2}+\left\|\nabla \theta_{z}\right\|_{L^{2}}^{2}+1\right) .
\end{aligned}
$$

From (25), Gronwall's inequality, (10), and (18), we know that $(u, \theta) \in L^{\infty}\left(0, T ; H^{1}\left(\mathbb{R}^{3}\right)\right)$. Thus $(u, \theta)$ can be extended smoothly beyond $t=T$. We have completed the proof of Theorem 1 .

Acknowledgements: The authors would like to thank the referee for their pertinent comments and advice. This work was supported in part by the NNSF of China (Grant No. 11101144) and Research Initiation Project for Highlevel Talents (201031) of North China University of Water Resources and Electric Power.

\section{REFERENCES}

1. Pedlosky J (1987) Geophysical Fluid Dynamics, Springer Verlag, New York.

2. Chae D (2006) Global regularity for the 2D Boussinesq equations with partial viscosity terms. Adv Math 203, 497-13.

3. Fan J, Zhou Y (2009) A note on regularity criterion for the 3D Boussinesq system with partial viscosity. Appl Math Lett 22, 802-5.

4. Xiang Z (2011) The regularity criterion of the weak solution to the $3 \mathrm{D}$ viscous Boussinesq equations in Besov spaces. Math Meth Appl Sci 34, 360-72.

5. Ishimura N, Morimoto H (1999) Remarks on the blow up criterion for the 3D Boussinesq equations. Math Model Meth Appl Sci 9, 1323-32.

6. Qin Y, Yang X, Wang Y, Liu X (2012) Blow-up criteria of smooth solutions to the 3D Boussinesq equations. Math Meth Appl Sci 35, 278-85.

7. Qiu H, Du Y, Yao Z (2010) A blow-up criterion for 3D Boussinesq equations in Besov spaces. Nonlin Anal 73, 806-15.

8. Leray J (1934) Sur le mouvement d'un liquide visqueux emplissant l'espace. Acta Math 63, 183-48.

9. Hopf E (1950) Über die Anfangswertaufgabe für die hydrodynamischen Grundgleichungen. Math Nachr $\mathbf{4}$, 213-31.

10. Serrin J (1962) On the interior regularity of weak solutions of the Navier-Stokes equations. Arch Ration Mech Anal 9, 187-95.

11. Struwe M (1988) On partial regularity results for the Navier-Stokes equations. Comm Pure Appl Math 41, 437-58.

12. He C (2002) New sufficient conditions for regularity of solutions to the Navier-Stokes equations. Adv Math Sci Appl 12, 535-48.

13. Kozono H, Yatsu N (2003) Extension criterion via twocomponents of vorticity on strong solution to the $3 \mathrm{D}$ Navier-Stokes equations. Math Z 246, 55-68.

14. Kukavica I, Ziane M (2007) Navier-Stokes equations with regularity in one direction. J Math Phys 48, 065203.

15. Zhang Z, Chen Q (2005) Regularity criterion via two components of vorticity on weak solutions to the Navier-Stokes equations in $\mathbb{R}^{3}$. J Differ Equat 216, 470-81.

16. Zhou Y (2006) On regularity criteria in terms of pressure for the Navier-Stokes equations in $\mathbb{R}^{3}$. Proc Am Math Soc 134, 149-56.

17. Zhou Y (2006) On a regularity criterion in terms of the gradient of pressure for the Navier-Stokes equations in $\mathbb{R}^{N}$. Z Angew Math Phys 57, 384-92.

18. Fan J, Ozawa T (2008) Regularity criterion for weak solutions to the Navier-Stokes equations in terms of the gradient of the pressure. J Inequal Appl 2008, 412678.

19. Zhou Y, Gala S (2009) Logarithmically improved regularity criteria for the Navier-Stokes equations in 
multiplier spaces. J Math Anal Appl 356, 498-501.

20. Fan J, Jiang S, Nakamura G, Zhou Y (2011) Logarithmically improved regularity criteria for the NavierStokes and MHD equations. J Math Fluid Mech 13, 557-71.

21. Adams R (1975) Sobolev Spaces, Academic Press, New York.

22. Cao C, Wu J (2010) Two regularity criteria for the 3D MHD equations. J Differ Equat 248, 2263-74.

23. Wang Y, Chen Z (2011) Regularity criterion for weak solution to the 3D micropolar fluid equations. $J$ Appl Math 2011, 456547. 